\title{
Study on Degradation Law and Related Mechanism of Mechanical Properties of Internal Reinforcement of RC Bridge After Uneven Corrosion
}

\author{
Hong-Yu Lu ${ }^{1}$, Man Zhou ${ }^{1}$, Bao-Hong Hao*1, Jian-Yu Pan ${ }^{1}$, Ding Zeng ${ }^{2}$ \\ ${ }^{1}$ Department of Mechanical Engineering, Beijing Institute of Petrochemical Technology, Beijing, China. \\ ${ }^{2}$ Research Institute of Highway Ministry of Transport, Ministry of Transport of the People's Republic of China, Beijing, China.
}

*Corresponding Author: Bao-Hong Hao, email: haobaohong@bipt.edu.cn

\section{Abstract}

In order to explore the corrosion process of the internal reinforcement of the bridge under the actual working conditions. In this paper, the accelerated stress corrosion test of HRB400e steel bar was carried out by full immersion method in laboratory, and the constant tensile stress was applied to the experimental steel bar in the form of constant moment beam specimen. After the test, the mechanical properties and microstructure of the steel bar were tested, and the experimental model was analyzed by finite element simulation. The experimental results show that with the increase of load, the corrosion degree of reinforcement increases gradually, and the number of pitting holes increases obviously. Microcracks lead to transgranular failure of metal lattice, which makes the microstructure more loose. The mechanical properties of steel bar have different degrees of degradation after rust, and the degradation of elongation is the most obvious. The results of finite element simulation are similar to the actual experiment, and there are obvious pitting pits at the stress concentration.

Keywords

Bridge Reinforcement; Non-uniform Corrosion; Mechanical Property Degradation; Metallographic Analysis; Finite Element Simulation

\section{INTRODUCTION}

In recent years, frequent bridge accidents have become one of the pain points of society. Due to steel structure corrosion, inadequate daily maintenance and management, severe overloading of vehicles, fatigue use and other reasons, there are many potential hazards to the safety of bridges, so that the actual life of reinforced concrete 
bridges after being put into service is far less than the theoretical life. If the bridge suddenly collapses, its loss and impact will be difficult to estimate. There are many factors affecting the actual service life of the bridge, and the relationships are complex and interactive, including corrosion of internal reinforcement, actual working load of the bridge and local climatic conditions. Among them, the main factor is that the mechanical properties of inner reinforcement greatly decrease after corrosion.

In the aspect of bridge reinforcement corrosion research: some scholars have established corrosion models under different corrosion states, and studied the degradation law of mechanical properties of corroded reinforcement through numerical simulation of experiments ${ }^{[1]}$. Some scholars have made small reinforced concrete specimens immersed in sodium chloride solution, and studied the corrosion effect of different sodium chloride concentrations on reinforcement in concrete ${ }^{[2]}$, Some researchers made reinforced concrete test beams and studied the influence of steel corrosion on the bearing capacity of beams ${ }^{[3-5]}$.

A lot of practices have proved that ${ }^{[6-11]}$, the corrosion of steel bars will result in the reduction of effective section area, the expansion and cracking of protective layer, and the consequent dramatic decrease of mechanical properties of concrete, which are the most important factors affecting the durability and service life of steel-concrete structures. Especially when the corrosion is serious to a certain extent, it will also form "local corrosion", which will lead to the disappearance of yield platform and material embrittlement, which will become a potential crisis for the overall collapse of the bridge. Therefore, it is of great significance and economic value to predict the service life of the bridge, master the actual situation of the operation of the bridge, timely and scientific maintenance.

\section{TEST MATERIALS AND METHODS}

The reinforcement bars in reinforced concrete bridge arise corrosion under bearing certain load, but little study on the corrosion problem of the bearing steel has been reported. In this paper, in order to explore the basic rules of the corrosion of stressed reinforcement bars, and objectively understand the present situation of existing reinforced concrete bridges, to provide a basis for objectively understanding the current situation of existing reinforced concrete bridges, scientifically manage and maintain bridges, and provide basis for more accurate prediction of bridges durability. Different tensile stresses are applied to reinforcement bars, and the corrosion problem of stressed steel bars is studied by exploratory tests.

In this experiment, the full immersion method was used to carry out accelerated stress corrosion test on the test reinforcement in the laboratory. The corrosion test pool was made of engineering plastic plate, and the corrosion medium was dilute hydrochloric acid solution. The $\mathrm{pH}$ value of the corrosion environment was controlled at about 3.0. HRB400e reinforcement is selected as the test reinforcement, and the specimen is made into a length of $1.2 \mathrm{~m}$ and a diameter of $22 \mathrm{~mm}$. The loading equipment adopts the form of constant moment beam specimen combination, and the loading frame applies constant tensile stress to the experimental steel bar. The loading frame is made of $\mathrm{H}$-shaped steel and is blackened to ensure that only minimal deformation occurs during the experiment, which will not affect the experiment. In order to avoid the influence of direct contact between the specimen and the loading frame on the electrochemical measurement data, insulation measures are taken. Accelerated corrosion tests (stress levels of $0 \mathrm{MPa}, 33 \mathrm{MPa}, 67 \mathrm{MPa}, 100 \mathrm{MPa}, 120 \mathrm{MPa}, 150 \mathrm{MPa}$ ) were carried out on steel bars after different prestressing forces were applied on the reinforcement frame.Each stress level consists of 4 test samples totaling 26 .

Table 1. Standard values for experimental reinforcement strength

\begin{tabular}{|c|c|c|c|}
\hline Metal brand & Nominal diameter(mm) & Standard value of yield strength(MPa) & Standard values for ultimate strength(MPa) \\
\hline HRB400e & $6 \sim 50$ & 400 & 540 \\
\hline
\end{tabular}




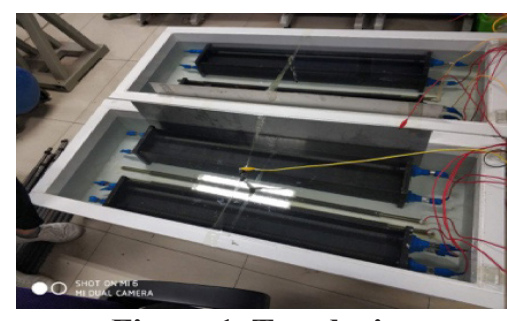

Figure 1. Test devices

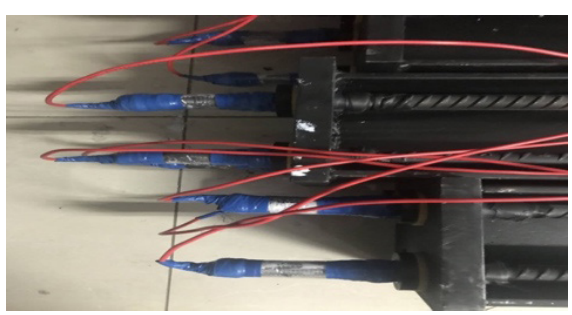

Figure 2. Welding lead

In this study, the corrosion parameters of stressed steel bar were measured by cs $310 \mathrm{~h}$ electrochemical workstation. The workstation was a conventional three electrode system, the auxiliary electrode was saturated calomel electrode, and the auxiliary electrode was 304 stainless steel (size: $1100 \times 350 \mathrm{~mm}$ ). The area of auxiliary electrode was much larger than the surface area of steel bar, and no reaction occurred during the experiment. The mechanical properties of the specimens after accelerated corrosion were tested. The test loading is controlled by the displacement speed of the specimen. According to the standard of tensile test, the actual displacement speed is taken as $10 \mathrm{~mm} / \mathrm{min}$. After the mechanical property test, the steel bar specimen was cut off to make metallographic specimen and observed with 200 times metallographic microscope.

\section{ANALYSIS OF EXPERIMENTAL RESULTS AND RELATED MECHANISM}

\subsection{Analysis of Electrochemical Results}

Figure 3 shows the polarization curve analysis of reinforcement with different stress levels under the same corrosion environment. It can be seen from the figure that with the increase of stress level, the polarization curve moves downward. The polarization curve fitted by potentiodynamic scanning shifts to the lower right, and the corrosion is more serious. The corrosion conditions of $33 \mathrm{MPa}$ and $67 \mathrm{MPa}$ are similar, while the corrosion rates of $100 \mathrm{MPa}$ and $150 \mathrm{MPa}$ are obviously different. The line moves more downward. With the increase of stress level, the corrosion rate increases, and the corrosion rate increases after $100 \mathrm{MPa}$.

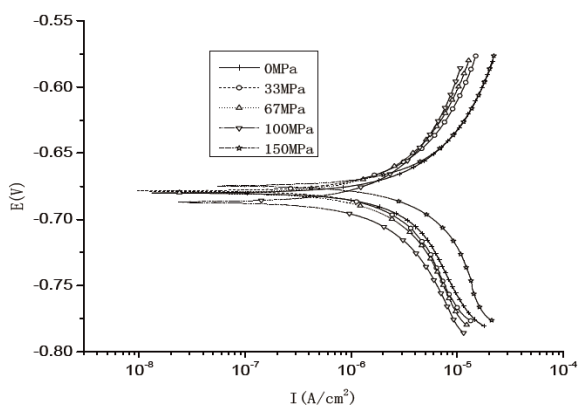

Figure 3. Polarization curves under different stress states

\subsection{Mechanical Property Test and Microstructure Analysis of Metal}

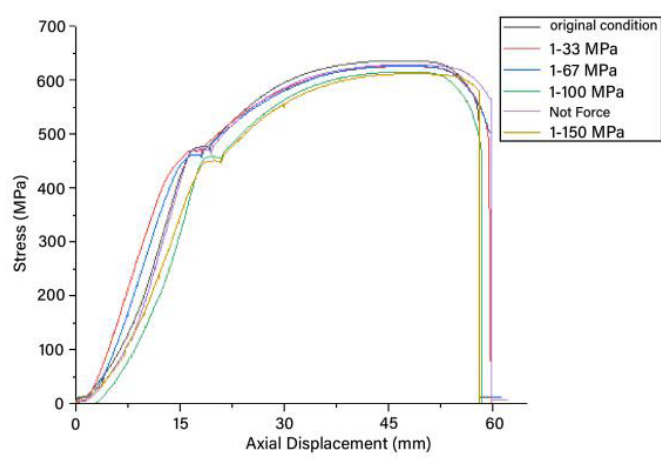

Figure 4. Mechanical property test

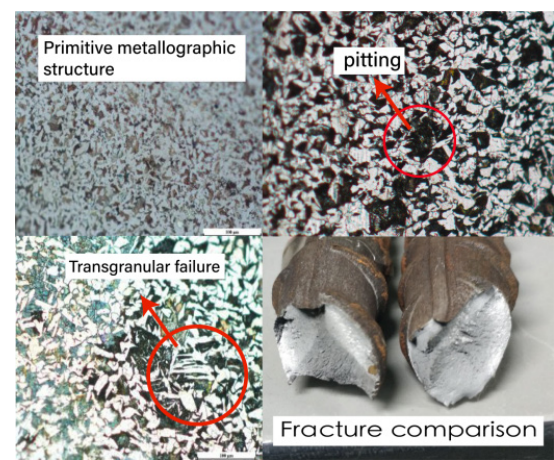

Figure 5. Metallographic test results 
The experimental results of mechanical properties show that with the increase of the stress state of the reinforcement, the yield platform in the stress-strain curve has an obvious downward trend, and the yield platform gradually shortens. With the increase of the pre-stress, the corrosion is serious, the necking phenomenon becomes not obvious, and the plastic deformation decreases, showing brittle fracture ${ }^{[12]}$.

With the increase of prestressing force, the metal crystal structure of the steel bar has changed obviously, and the structure defects such as pitting pit and transgranular damage appear gradually in the seriously corroded area, which will lead to the increase of the spacing of the metal lattice structure and make the structure become loose and unstable. These defects will seriously reduce its mechanical properties.

\subsection{Multi Physical Field Simulation Analysis}
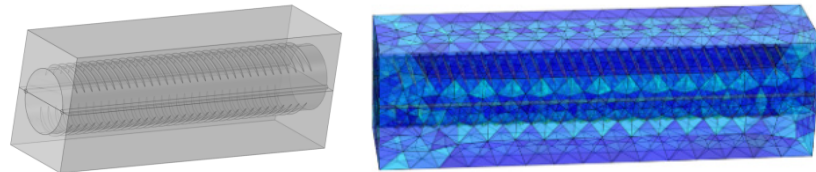

Figure 6. 3D geometric model and grid division

In order to explore the influence of stress on steel bar corrosion, COMSOL simulation is carried out according to the actual test conditions and contents. Coupling solid mechanics with secondary current distribution, the outer surface of the reinforcement is set as the electrochemical reaction electrode surface, and the conductivity of electrolyte is $2.5 \mathrm{~S} / \mathrm{m}$.In solid mechanics, one end of the reinforcing bar is fixed and the other end is constrained by fixed constraints $\mathrm{P}$ of $0,33 \mathrm{mpa}, 67 \mathrm{MPa}, 100 \mathrm{MPa}, 120 \mathrm{MPa}$ and $150 \mathrm{MPa}$, which are in accordance with the actual situation.Observe the stress distribution on the surface of reinforcement.

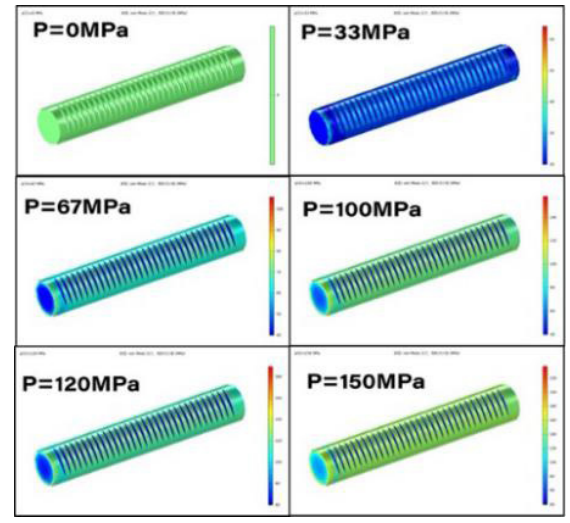

Figure 7. Stress

distribution steel surface

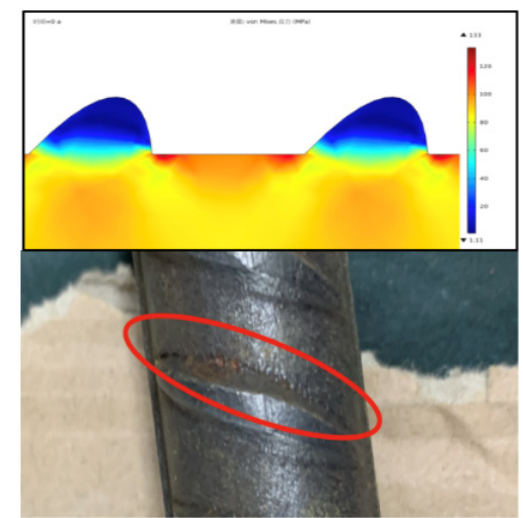

Figure 8. The simulation results are on compared with the actual results

By the diagram can be seen, not when the applied stress uniform stress distribution for steel surface controlled trials, when applying different reinforcement stress, the stress is not the same size, on the surface of the stress concentration in the steel surface between the rib, but due to the uneven distribution of rebar structure, lead to the stress distribution is uneven.

To explore the internal stress distribution of steel bar, the above solid three-dimensional model is taken from the $1 / 4$ section of ribbed strip for two- dimensional modeling. It can be seen from the diagram that because of the sharp change in the shape of the junction between steel bar and steel bar surface, and in the production and processing of steel bar, the position is the most uneven distribution of the structure, so most of the stress is concentrated here. It can also be seen that the stress value of the surface increases with the increase of the applied load. When the applied stress is $150 \mathrm{MPa}$, the highest stress value on the surface has reached $237 \mathrm{Mpa}$. 


\section{CONCLUSION}

Main conclusions can be obtained as follows:

(1) In the process of steel corrosion, the corrosion rate of steel bars increased with the increase of prestress. However, the dissolution of anode under the action of corrosive medium lead to anodic polarization and passivation near the polarization zone. Moreover, a large number of corrosion products accumulate on the surface of the steel bar, which hindered the contact of corrosion ions with metal and reduced the corrosion rate in the middle stage of corrosion. As time goes by, the corrosion further intensified, and the corrosion rate increased under the combined action of stress and corrosion ions.

(2) Pitting corrosion and microcracks appear in the metal lattice under the combined action of stress and corrosion ions, which caused microscopic crystal structure to be loose and the stability of the metal decreases. The macroscopic manifestation is that with the increase of prestress, the fracture mode turned from plastic fracture to brittle fracture. Therefore, the bridge needs to be maintained and strengthened before the crystal structure inside the bridge reinforcement changes.

(3) COMSOL analysis showed that the uniaxial tensile specimen was directly applied, the magnitude of applied stress can be precisely controlled and the stress map was simple and uniform. The most serious location where the corrosion ribbed steel bars occurred is at the connection between ribs and surface of steel bars. Obvious pitting corrosion and degradation of mechanical properties were obvious. This research provides a scientific basis for the study and judgment, maintenance and life prediction of actual conditions of prestressed concrete bridges, and has practical significance and practical value for improving the durability of bridges.

\section{ACKNOWLEDGEMENT}

Supported by a special fund project for:

1. Project name: The construction of random model of corrosion degree focusing on material heterogeneity and the prediction of bridge durability considering the time variation of real bridge.

2. Project name: Entrepreneurship training plan for College Students, Project name, Bridge accounting test paper -- Creating a new digital model of bridge security Check, Project No. 2020100170043.

\section{REFERENCE}

[1] Ouyang Xiangsen, Zhang Yanfang, Zou Hongbo. Degradation analysis of mechanical properties of corroded steel bars based on numerical simulation [J]. Journal of Hunan Institute of Engineering (NATURAL SCIENCE EDITION), 2018, 88 (02): 68-72

[2] He Yong, Tang Xianxi, Sun Shuaihu,et al. Experimental study on corrosion resistance of reinforced concrete bridge based on electrochemical corrosion[J] China concrete and cement products, 2018(9):1-6

[3] Zhang Jianren, Zhang Kebo, Peng Hui, et al. Calculation Method of Normal Section Flexural Capacity of Corroded Reinforced Concrete Rectangular Beams[J]. China Journal of Highway and Transport, 2009,22(3):45-51

[4] Fares J,Riyad S A. Residual flexural strength of corroded reinforced concrete beams[J]. Engineering Structures,2016,119( 7):198-216

[5] Yang Xiaoming, Cheng Chunhui, Chen He. Condition assessment of corroded reinforced concrete component considering Degradation of Load Carrying Capacity[J]. Bulletin of the Chinese ceramic society, 2018,37(3):837-843

[6] Liang Fan, Xiao Tan, Qinghua Zhang, et al. Monitoring corrosion of steel bars in reinforced concrete based on helix strains measured from a distributed fiber optic sensor[J]. Engineering Structures, 2020, 204: 1-10. 
[7] Zhang Bai, Zhu Hong, Chen Jun, Yang Ou. Influence of specimen dimensions and reinforcement corrosion on bond performance of steel bars in concrete[J]. Advances in Structural Engineering, 2020. 1(10): 10-17.

[8] Antonino R, Nino S, Francesco T. Failure analysis of corroded RC beams subjected toshear-flexural actions[J]. Engineering Failure Analysis, 2018,93(8):26-37.

[9] Berrocal C G, Fernandez I, Lundgren K, et al. Corrosion-induced cracking and bond behaviour of corroded reinforcement bars in SFRC[J]. Composites Part B: Engineering, 2017, 113: 123-137.

[10] Ahsana P V, Rao K B, Anoop M B. Time-variant reliability analysis of RC bridge girders subjected to corrosion - shear limit state[J]. Structural Concrete, 2016, 17(2): 162-174.

[11] Paul Jurišić, Joško Parunov. Influence of corrosion-related degradation of mechanical properties of shipbuilding steel on collapse strength of plates and stiffened panels[J]. Towards Green Marine Technology and Transport, 2015, 427-432.

[12] Ding Zeng, Hong Yu Lu, Bao Hong Hao, et al. Experimental Study and Mechanism on the Corrosion of Stressed Reinforcement Bars. 2020, 5973: 109-115. 